\title{
Real-world Effectiveness of Liraglutide vs. Sitagliptin Among Older Patients with Type 2 Diabetes Enrolled in a Medicare Advantage Prescription Drug Plan: A Retrospective Observational Study
}

\author{
Tam Dang-Tan • Pravin S. Kamble • Yunus Meah • Cory Gamble • \\ Rahul Ganguly · Libby Horter
}

Received: August 19, 2019 / Published online: December 9, 2019

(C) The Author(s) 2019

\section{ABSTRACT}

Introduction: Liraglutide and sitagliptin were compared on glycemic control and all-cause healthcare costs over a 1-year period among older adults with type 2 diabetes (65-89 years) enrolled in a national Medicare Advantage Prescription Drug health plan.

Methods: This was a retrospective study in which the index date was the first prescription fill for liraglutide or sitagliptin between 25 January 2010 and 31 December 2014. Post-index treatment persistence and glycosylated hemoglobin $\left(\mathrm{HbA} 1_{\mathrm{c}}\right)$ at baseline and 1 year ( \pm 90 days) post-index date were required. Patients were excluded if their record included use of insulin during the baseline period. Inverse probability of treatment weighting using stabilized weights was employed with final covariate adjusted regression modeling to

Enhanced Digital Features To view enhanced digital features for this article go to https://doi.org/10.6084/ m9.figshare.10760369.

T. Dang-Tan $(\bowtie) \cdot$ C. Gamble $\cdot$ R. Ganguly

Novo Nordisk Inc., Plainsboro, NJ, USA

e-mail: tadt@novonordisk.com

P. S. Kamble · L. Horter

Humana Healthcare Research, Inc., Louisville, KY, USA

Y. Meah

Humana Inc., Louisville, KY, USA estimate the primary outcome (mean change in $\mathrm{HbA} 1_{\mathrm{c}}$ ) and secondary outcomes (achieving glycemic goal and costs), each at 1 -year postindex date.

Results: Overall, 3056 patients met the selection criteria, of whom 218 filled prescriptions for liraglutide and 2838 for sitagliptin. Adjusted mean change in $\mathrm{HbA}_{\mathrm{c}}$ at 1 year post-index was -0.42 with liraglutide versus -0.12 with sitagliptin $(P=0.0012)$. Adjusted odds of achieving the treatment goals of $\mathrm{HbA} 1_{c}<7 \%$ and achieving an $\mathrm{HbA}_{\mathrm{c}}$ reduction of $\geq 1 \%$ were higher for those on liraglutide than for those on sitagliptin $(1.68,95 \%$ confidence interval $[\mathrm{CI}] 1.25-2.24$ and $1.76,95 \%$ CI 1.31-2.36), respectively. Total healthcare costs in those achieving an $\mathrm{HbA}_{\mathrm{c}}$ of $<7 \%$ were not significantly different between treatment groups but were higher within the liraglutide group for those achieving an $\mathrm{HbA} 1_{\mathrm{c}}<8 \%$.

Conclusions: When compared to sitagliptin, liraglutide was associated with greater achievement of an $\mathrm{HbA} 1_{\mathrm{c}}<7 \%$ over a 1 -year period in an older population. This finding was not associated with a statistically significant increase in all-cause total healthcare costs, although costs were slightly higher in the liraglutide group than in the sitagliptin group.

Keywords: Clinical outcomes; Liraglutide; Older adults; Sitagliptin; Type 2 diabetes 


\section{Key Summary Points}

Why carry out this study?

Type 2 diabetes (T2D) is a rising health concern in the USA, particularly within an aging population.

Despite the importance of effectively managing T2D in older adults, this patient population has often been excluded from randomized clinical trials, and limited data exist on this older population regarding real-world outcomes related to different glucose-lowering therapies.

\section{What was learned from the study?}

Liraglutide and sitagliptin were compared on glycemic control and all-cause healthcare costs over a 1-year period among older adults with T2D enrolled in a national Medicare Advantage Prescription Drug health plan.

When compared to sitagliptin use, liraglutide use was associated with greater achievement of an glycosylated hemoglobin level of $<7 \%$ over a 1 -year period in an older population.

This finding was not associated with an increase in all-cause total healthcare costs.

\section{INTRODUCTION}

Type 2 diabetes (T2D) is a rising health concern in the USA, particularly within an aging population. In 2015, $9.4 \%$ of the total US population was estimated to have diabetes; this number increased to $17.0 \%$ when this analysis was restricted to adults aged 45-64 years only and to $25.2 \%$ among adults aged 65 years or older [1]. This number is expected to continue to rise due to the aging US population and increased life expectancy of people with diabetes. Older diabetic patients have a higher risk for diabetesrelated complications, including microvascular and macrovascular damage and hypoglycemia, and therefore present significant challenges in achieving strict glycemic control and constitute a growing burden on the US healthcare system [2-4].

Despite the importance of effectively managing T2D in older adults, this patient population has often been excluded from randomized clinical trials, and limited data exist on this older population regarding real-world outcomes related to different glucose-lowering therapies [4]. The class of incretin-based therapies, including glucagon-like peptide-1 receptor agonists (GLP-1 RAs) and dipeptidyl peptidase 4 (DPP-4) inhibitors, may allow for improved control of hyperglycemia and offers important advantages to an older population (i.e., minimal risk for hypoglycemia, weight loss, and lower risk for cardiovascular disease associated with GLP-1 RAs) [5]. Previous studies have found that the GLP-1 analogue liraglutide provides sustained glycosylated hemoglobin $\left(\mathrm{HbA1} 1_{\mathrm{c}}\right)$ reduction, achievement of specific $\mathrm{HbA} 1_{\mathrm{c}}$ goals, and weight loss when compared to the DPP-4 inhibitor sitagliptin [6-12]. Additionally, retrospective observational studies have demonstrated the cost-effectiveness of liraglutide when compared within and between antidiabetic drug classes; however, cost-effectiveness has not been specifically explored in a T2D population aged 65 years and older [11, 13-16].

The aim of the current study was to compare liraglutide with sitagliptin in achieving glycemic control among older people with T2D enrolled in a Medicare Advantage Prescription Drug (MAPD) plan. This was accomplished by evaluating mean change in $\mathrm{HbA} 1_{\mathrm{c}}$, mean reduction in $\mathrm{HbA}_{\mathrm{c}}$ of $\geq 1 \%$, and the percentage of patients achieving the treatment goals of $\mathrm{HbA}_{\mathrm{c}}<7 \%$ and $\mathrm{HbA}_{\mathrm{c}}<8 \%$ over a 1 -year period. The analysis also compared all-cause healthcare costs (pharmacy and medical combined) in this population. This information may be important when considering real-world treatment in the growing older population with T2D in the USA. 


\section{MATERIALS AND METHODS}

\section{Data Source}

This was a retrospective and observational study. The Humana Research Database (Humana, Louisville, KY), which contains administrative claims data for individuals enrolled in Humana's fully insured commercial and medicare plans was used to compare clinical and cost outcomes between patients treated with liraglutide and those treated with sitagliptin. The database included medical, pharmacy, and laboratory claims of individuals with T2D enrolled in a MAPD plan for the period of 25 July 2009-30 March 2016. The research protocol associated with the manuscript was reviewed and approved as a minimal risk study by Schulman IRB, an independent institutional review board, which determined that the study met the criteria for a waiver of informed consent and waiver of authorization as set forth by the code of federal regulations.

\section{Sample Selection}

Analysis included patients (age 65-89 years at index date) who received their first prescription for liraglutide or sitagliptin between 25 January 2010, and 31 December 2014. Patients were also required to have continuous MAPD plan membership throughout the study period, including enrollment for at least 6 months pre-index date and 15 months post-index date (Fig. 1).
Additional inclusion criteria were: evidence of T2D (ICD-9 [International Classification of Diseases, Ninth Revision, Clinical Modification] codes $250 . x 0$ or $250 . x 2$ in any position on $\geq 1$ outpatient, acute inpatient, or emergency department [ED] claim in the study period); post-index persistence (defined as having no gaps in treatment of $\geq 60$ days in the 365 day post -index treatment period), and available baseline and 1-year follow-up $\mathrm{HbA} 1_{c}$ values. Patients were excluded if their record contained evidence of type 1 diabetes mellitus, DPP-4 inhibitor use, or sodium glucose cotransporter 2 inhibitor use in the baseline period, and not meeting the requirement of persistence on medication for 1 year. To minimize possible biases and reduce baseline differences between the two groups, patients were also excluded if they had a record of insulin use in the baseline period.

\section{Study Measures}

Patient information included demographics, such as age, gender, and race/ethnicity, and baseline clinical characteristics, such as DeyoCharleston Comorbidity Index, Diabetes Complications Severity Index (DCSI), HbA1 c level, comorbidities, and the use of antidiabetic medications.

The primary outcome measure was mean change in $\mathrm{HbA}_{\mathrm{c}}$ from baseline to the 1 year ( \pm 90 days) follow-up. If more than one HbA1c result was available, the test result closest to the index date was used for the baseline measure,

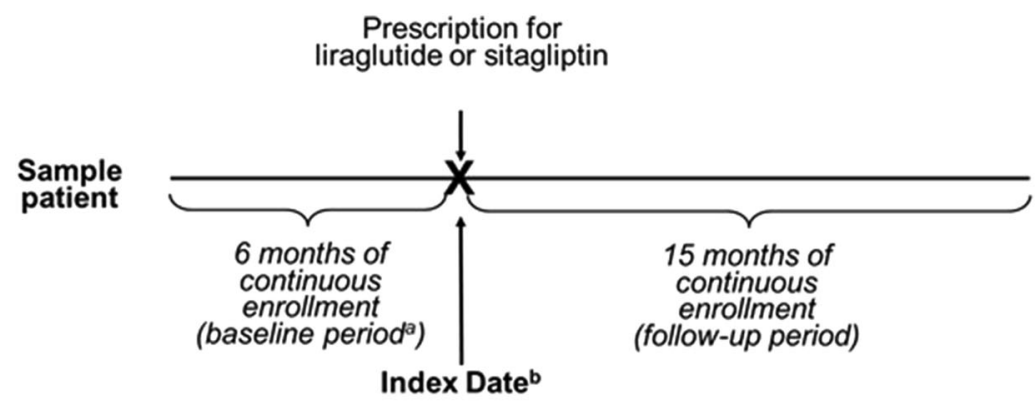

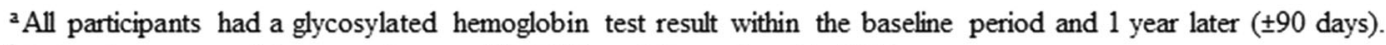

${ }^{b}$ Index date occurred between January 25, 2010, and December 31, 2014.

Fig. 1 Patient sample selection 
and the test result closest to 1 year from the index date was used for the 1-year follow-up. Secondary outcome measures were percentage of patients achieving a mean reduction in $\mathrm{HbA} 1_{\mathrm{c}}$ of $\geq 1 \%$, percentage of patients achieving the treatment goals of $\mathrm{HbA} 1_{\mathrm{c}}<7 \%$ and $\mathrm{HbA} 1_{\mathrm{c}}<8 \%$, and percentage of patients achieving these treatment goals with no reported hypoglycemia. Total healthcare costs (allcause pharmacy and medical) in patients achieving these goals with no reported hypoglycemia were also measured. Costs were calculated separately for inpatient hospital, ED, physician office visits, nursing facility, other outpatient encounters, and pharmacy services, and were based on the total amount allowed by the healthcare plan for a given procedure or healthcare encounter. To mitigate the potential for underestimating costs of services provided under capitated arrangements, costs for these services were imputed at the service-line level. Capitated costs were assigned the median value (allowed amount) from non-capitated fee-forservice claims matched by procedure and payment level. Payment level was derived from the source of billing (facility or professional) and the place of service (physician office or facility), similar to the Medicare prospective payment systems. Costs were adjusted to the 2015 value based on the Consumer Price Index Medical Component [17]. Due to a low number of postindex hypoglycemic events, 'no hypoglycemia' was not included in the composite outcome.

\section{Statistical Analysis}

Descriptive analyses included demographics, baseline characteristics, and primary and secondary outcomes and were reported as number with percentage, mean with standard deviation, or median with interquartile range. Changes in $\mathrm{HbA} 1_{\mathrm{c}}$ were analyzed by the $t$ test, the proportions of patients achieving treatment goals were analyzed using the Chi-square test, and costs of achieving treatment goals were analyzed using the Wilcoxon rank sum test. $P$ values of $<0.05$ were considered to be statistically significant.

Glycemic control outcomes were modeled using linear regression (mean change in $\mathrm{HbA}_{\mathrm{c}}$ ) and multiple logistic regression (proportions of patients achieving $\mathrm{HbA}_{\mathrm{c}}$ treatment goals). Rigorous weighting methods (inverse probability of treatment weighting [IPTW] using stabilized weights) [18] were used in adjusted analyses to reduce bias and measured confounding attributed to the nature of the retrospective study design. For the primary outcome, the sample size requirement was estimated to be 140 older patients with T2D in the liraglutide group and 1258 in the sitagliptin group, based on group weights of 10 vs. 90\%, respectively, and the detection of a mean change in $\mathrm{HbA} 1_{\mathrm{c}}$ of 0.4 using a two-sided test with $\alpha=0.05$ and power $1-\beta=0.80$. To ensure that these statistical methods had achieved adequate balance, baseline covariates were compared between treatment groups by calculating standardized differences. These baseline covariates were considered to be balanced across patient groups if standardized differences were $<0.10$ (see Table 1). After weighting, balance was achieved for most variables, except for four covariates (gender, race, health plan type, and level of prior antidiabetic medication use). These four variables were included as independent variables in the final IPTW regression models, along with post-index antidiabetic treatment additions. The final model to detect the difference in change in mean $\mathrm{HbA}_{\mathrm{c}}$ between the treatment groups was adequately powered. Estimated outcomes of glycemic control were reported as odds ratios with 95\% confidence intervals (CIs).

Estimated all-cause total cost data (pharmacy and medical) were analyzed using generalized linear models based on a log link and gamma distribution, with and without covariate adjustment.

\section{RESULTS}

Overall, 3056 patients met the criteria of persistence on index treatment and had $\mathrm{HbA}_{\mathrm{c}}$ results available within the baseline period and 1 year later (Fig. 2). Within this study population, $218(7.1 \%)$ patients were treated with liraglutide and 2838 (92.9\%) patients were treated with sitagliptin. 
Table 1 Assessment of balance between treatment groups between observed and weighted

\begin{tabular}{|c|c|c|c|c|c|c|}
\hline \multirow[t]{2}{*}{ Characteristic } & \multicolumn{3}{|l|}{ Observed } & \multicolumn{3}{|c|}{ Weighted (stabilized IPTW) } \\
\hline & $\begin{array}{l}\text { Liraglutide } \\
\text { cohort } \\
(n=218)\end{array}$ & $\begin{array}{l}\text { Sitagliptin } \\
\text { cohort } \\
(n=2838)\end{array}$ & $\begin{array}{l}\text { Standardized } \\
\text { difference }\end{array}$ & $\begin{array}{l}\text { Liraglutide } \\
\text { cohort } \\
(n=218)\end{array}$ & $\begin{array}{l}\text { Sitagliptin } \\
\text { cohort } \\
(n=2838)\end{array}$ & $\begin{array}{l}\text { Standardized } \\
\text { difference }\end{array}$ \\
\hline Age, years, mean (SD) & $70.5(4.7)$ & $73.2(5.9)$ & 0.5187 & $70.5(4.7)$ & $73.2(5.9)$ & 0.0829 \\
\hline \multicolumn{7}{|l|}{ Gender, $n(\%)$} \\
\hline Female & $115(52.8)$ & $1451(51.1)$ & & $115(52.8)$ & $1451(51.1)$ & \\
\hline Male & $103(47.3)$ & $1387(48.9)$ & 0.0325 & $103(47.3)$ & $1387(48.9)$ & 0.137 \\
\hline \multicolumn{7}{|l|}{ Geographic region, $n$ (\%) } \\
\hline Northeast & $-{ }^{\mathrm{a}}$ & $28(1.0)$ & 0.0071 & $-{ }^{\mathrm{a}}$ & $28(0.99)$ & 0.0132 \\
\hline Midwest & $49(22.5)$ & $443(15.6)$ & 0.1756 & $49(22.5)$ & $443(15.6)$ & 0.049 \\
\hline South & $140(64.2)$ & $2079(73.3)$ & 0.1959 & $140(64.2)$ & $2079(73.3)$ & 0.0586 \\
\hline West & $27(12.4)$ & $288(10.2)$ & 0.0708 & $27(12.4)$ & $288(10.2)$ & 0.023 \\
\hline \multicolumn{7}{|l|}{ Race/ethnicity, $n$ (\%) } \\
\hline White & $202(92.7)$ & $2189(77.1)$ & 0.4442 & $202(92.7)$ & $2189(77.1)$ & 0.0544 \\
\hline Black & $-{ }^{\mathrm{a}}$ & $403(14.2)$ & 0.3545 & $-{ }^{\mathrm{a}}$ & $403(14.2)$ & 0.1014 \\
\hline Hispanic & $-{ }^{\mathrm{a}}$ & $104(3.7)$ & 0.1121 & $-{ }^{a}$ & $104(3.7)$ & 0.0385 \\
\hline Other & $-{ }^{\mathrm{a}}$ & $142(5.0)$ & 0.2075 & $-{ }^{a}$ & $142(5.0)$ & 0.0151 \\
\hline \multicolumn{7}{|c|}{ Healthcare plan type, $n(\%)$} \\
\hline $\mathrm{HMO}$ & $125(57.3)$ & $1712(60.3)$ & 0.0607 & $125(57.3)$ & $1712(60.3)$ & 0.0031 \\
\hline $\mathrm{PPO}$ & $75(34.4)$ & $794(28.0)$ & 0.139 & $75(34.4)$ & $794(28.0)$ & 0.0733 \\
\hline POS & $-{ }^{\mathrm{a}}$ & $39(1.4)$ & 0.0367 & $-{ }^{a}$ & $39(1.4)$ & 0.0464 \\
\hline FFS & $11(5.1)$ & $166(5.9)$ & 0.0354 & $11(5.1)$ & $166(5.9)$ & 0.007 \\
\hline Other & $-{ }^{\mathrm{a}}$ & $127(4.5)$ & 0.1847 & $-{ }^{a}$ & $127(4.5)$ & 0.2387 \\
\hline \multicolumn{7}{|c|}{ Healthcare plan characteristics, $n(\%)$} \\
\hline LIS status only & $17(7.8)$ & $174(6.1)$ & 0.0655 & $17(7.8)$ & $174(6.1)$ & 0.0765 \\
\hline Dual eligibility only & $-{ }^{\mathrm{a}}$ & $-{ }^{\mathrm{a}}$ & 0.0703 & $-{ }^{a}$ & $-{ }^{a}$ & 0.0678 \\
\hline $\begin{array}{l}\text { LIS status and dual } \\
\text { eligibility }\end{array}$ & $31(14.2)$ & $628(22.1)$ & 0.2062 & $31(14.2)$ & $628(22.1)$ & 0.0102 \\
\hline $\begin{array}{l}\text { Deyo-CC Index, } \\
\text { mean }(S D)\end{array}$ & $1.7(1.47)$ & $2.31(1.9)$ & 0.3558 & $1.7(1.5)$ & $2.31(1.9)$ & 0.022 \\
\hline DCSI, mean (SD) & $0.69(1.3)$ & $1.27(1.6)$ & 0.4028 & $0.69(1.3)$ & $1.27(1.6)$ & 0.0271 \\
\hline \multicolumn{7}{|c|}{ Presence of comorbidity: $n$ (\%) } \\
\hline $\begin{array}{l}\text { Cardiovascular } \\
\text { disease }\end{array}$ & $26(11.9)$ & $755(26.6)$ & 0.3788 & $26(11.9)$ & $755(26.6)$ & 0.0318 \\
\hline
\end{tabular}


Table 1 continued

\begin{tabular}{|c|c|c|c|c|c|c|}
\hline \multirow[t]{2}{*}{ Characteristic } & \multicolumn{3}{|l|}{ Observed } & \multicolumn{3}{|c|}{ Weighted (stabilized IPTW) } \\
\hline & $\begin{array}{l}\text { Liraglutide } \\
\text { cohort } \\
(n=218)\end{array}$ & $\begin{array}{l}\text { Sitagliptin } \\
\text { cohort } \\
(n=2838)\end{array}$ & $\begin{array}{l}\text { Standardized } \\
\text { difference }\end{array}$ & $\begin{array}{l}\text { Liraglutide } \\
\text { cohort } \\
(n=218)\end{array}$ & $\begin{array}{l}\text { Sitagliptin } \\
\text { cohort } \\
(n=2838)\end{array}$ & $\begin{array}{l}\text { Standardized } \\
\text { difference }\end{array}$ \\
\hline Nephropathy & $29(13.3)$ & $754(26.6)$ & 0.3367 & $29(13.3)$ & $754(26.6)$ & 0.005 \\
\hline Retinopathy & $-{ }^{a}$ & $116(4.1)$ & 0.0216 & $-{ }^{\mathrm{a}}$ & $116(4.09)$ & 0.0545 \\
\hline $\begin{array}{l}\text { Peripheral vascular } \\
\text { disease }\end{array}$ & $13(6.0)$ & $249(8.8)$ & 0.1077 & $13(6.0)$ & $249(8.8)$ & 0.0883 \\
\hline $\begin{array}{l}\text { Cerebrovascular } \\
\text { disease }\end{array}$ & $-{ }^{\mathrm{a}}$ & $94(3.3)$ & 0.0934 & $-{ }^{\mathrm{a}}$ & $94(3.3)$ & 0.0299 \\
\hline Neuropathy & $29(13.3)$ & $444(15.6)$ & 0.0666 & $29(13.3)$ & $444(15.6)$ & 0.092 \\
\hline Metabolic disease & $-{ }^{a}$ & $-{ }^{\mathrm{a}}$ & 0.0594 & $-{ }^{\mathrm{a}}$ & $-{ }^{a}$ & 0.0573 \\
\hline Obesity & $62(28.4)$ & $445(15.7)$ & 0.3114 & $62(28.4)$ & $445(15.7)$ & 0.0174 \\
\hline Hypoglycemia & $-{ }^{\mathrm{a}}$ & $132(4.7)$ & 0.0255 & $-{ }^{\mathrm{a}}$ & $132(4.7)$ & 0.0529 \\
\hline $\begin{array}{l}\text { Pre-index unique } \\
\text { medication counts, } \\
\text { mean }(S D)\end{array}$ & $10.66(3.9)$ & $10.74(4.2)$ & 0.0199 & $10.66(3.9)$ & $10.74(4.2)$ & 0.0634 \\
\hline $\begin{array}{l}\text { Pre-index prescription } \\
\text { fill, counts, mean } \\
\text { (SD) }\end{array}$ & $13.58(8.0)$ & $14.65(8.3)$ & 0.1307 & $13.58(8.0)$ & $14.65(8.3)$ & 0.0059 \\
\hline \multicolumn{7}{|c|}{ Utilization of antidiabetic medications during pre-index period: $n(\%)$} \\
\hline Biguanides & $166(76.2)$ & $2062(72.7)$ & 0.08 & $166(76.2)$ & $2062(72.7)$ & 0.0043 \\
\hline Sulfonylurea & $130(59.6)$ & $1811(63.8)$ & 0.0861 & $130(59.6)$ & $1811(63.8)$ & 0.073 \\
\hline Thalidozlinide & $39(17.9)$ & $489(17.2)$ & 0.0173 & $39(17.9)$ & $489(17.2)$ & 0.054 \\
\hline $\begin{array}{l}\text { Other antidiabetic } \\
\text { medication }\end{array}$ & $-{ }^{\mathrm{a}}$ & $70(2.5)$ & 0.0179 & $-{ }^{\mathrm{a}}$ & $70(2.5)$ & 0.0591 \\
\hline \multicolumn{7}{|c|}{ Pre-index level of antidiabetic therapy, $n(\%)$} \\
\hline No medication use & $12(5.5)$ & $217(7.7)$ & 0.0865 & $12(5.5)$ & $217(7.7)$ & 0.1229 \\
\hline $\begin{array}{l}1 \text { non-insulin } \\
\text { antidiabetic }\end{array}$ & $100(45.9)$ & $1211(42.7)$ & 0.0645 & $100(45.9)$ & $1211(42.7)$ & 0.0901 \\
\hline $\begin{array}{l}2 \text { non-insulin } \\
\text { antidiabetics }\end{array}$ & 87 (39.9) & $1221(43.0)$ & 0.0633 & $87(39.9)$ & $1221(43.0)$ & 0.0068 \\
\hline $\begin{array}{l}\geq 3 \text { non-insulin } \\
\text { antidiabetics }\end{array}$ & $19(8.7)$ & $189(6.7)$ & 0.0772 & $19(8.7)$ & $189(6.7)$ & 0.0508 \\
\hline $\begin{array}{l}\text { Pre-index } \mathrm{HbAl}_{\mathrm{c}} \\
\text { mean }(\mathrm{SD})\end{array}$ & $8.03(1.4)$ & $7.8(1.4)$ & 0.1714 & $8.03(1.4)$ & $7.8(1.4)$ & 0.0892 \\
\hline
\end{tabular}


Table 1 continued

\begin{tabular}{|c|c|c|c|c|c|c|}
\hline \multirow[t]{2}{*}{ Characteristic } & \multicolumn{3}{|l|}{ Observed } & \multicolumn{3}{|c|}{ Weighted (stabilized IPTW) } \\
\hline & $\begin{array}{l}\text { Liraglutide } \\
\text { cohort } \\
(n=218)\end{array}$ & $\begin{array}{l}\text { Sitagliptin } \\
\text { cohort } \\
(n=2838)\end{array}$ & $\begin{array}{l}\text { Standardized } \\
\text { difference }\end{array}$ & $\begin{array}{l}\text { Liraglutide } \\
\text { cohort } \\
(n=218)\end{array}$ & $\begin{array}{l}\text { Sitagliptin } \\
\text { cohort } \\
(n=2838)\end{array}$ & $\begin{array}{l}\text { Standardized } \\
\text { difference }\end{array}$ \\
\hline \multicolumn{7}{|c|}{ Prescribing physician specialty, $n$ (\%) } \\
\hline Primary care & $91(41.7)$ & $1094(38.6)$ & 0.0652 & $91(41.7)$ & $1094(38.6)$ & 0.0067 \\
\hline Endocrinology & $31(14.2)$ & $155(5.5)$ & 0.2973 & $31(14.2)$ & $155(5.5)$ & 0.0167 \\
\hline $\begin{array}{l}\text { Internal and family } \\
\text { medicine }\end{array}$ & $65(29.8)$ & $1299(45.8)$ & 0.3336 & $65(29.8)$ & $1299(45.8)$ & 0.0036 \\
\hline Other & $40(18.4)$ & $349(12.3)$ & 0.1686 & $40(18.4)$ & $349(12.3)$ & 0.0062 \\
\hline
\end{tabular}

DCSI Diabetes Complications Severity Index, Deyo-CC Deyo-Charlson Comorbidity Index, FFS fee for service, $H b A I_{c}$ glycosylated hemoglobin, $H M O$ health management organization, IPTW inverse probability of treatment weighting, LIS low income subsidy, POS point of service, $P P O$ preferred provider organization, $S D$ standard deviation

${ }^{a}$ Data suppressed to protect privacy

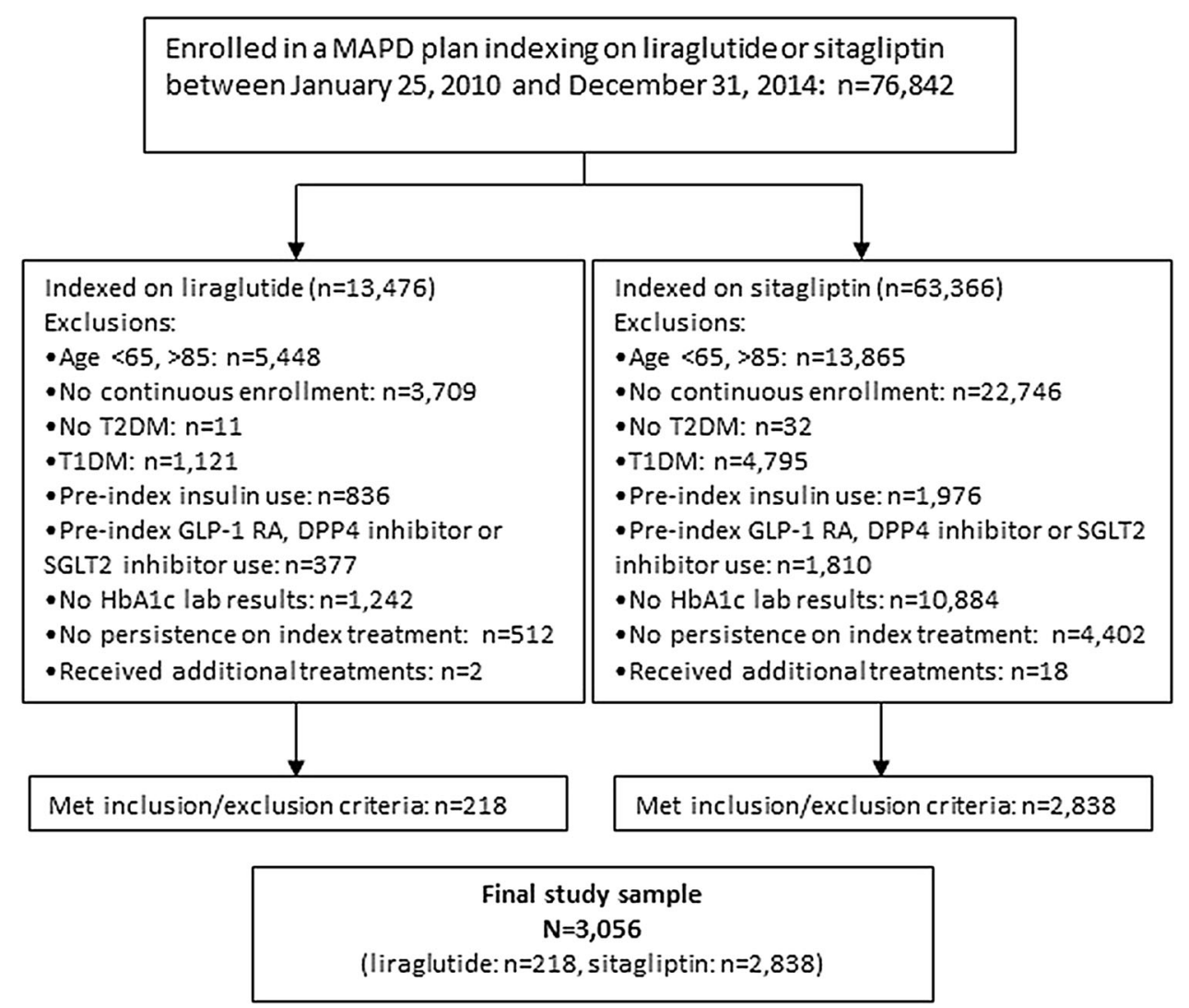

Fig. 2 Patient attrition. DDP-4 Dipeptidyl peptidase 4, $H b A 1_{c}$ glycosylated hemoglobin, MAPD Medicare Advantage Prescription Drug, GLP-1 RA glucagon-like peptide-1 receptor agonist, $S G L T 2$ sodium glucose cotransporter 2 inhibitor, $T 1 D M, T 2 D M$ type 1 , type 2 diabetes mellitus, respectively 
Table 2 Baseline characteristics of the liraglutide and sitagliptin cohorts

\begin{tabular}{|c|c|c|c|}
\hline Characteristic & $\begin{array}{l}\text { Liraglutide cohort } \\
(n=218)\end{array}$ & $\begin{array}{l}\text { Sitagliptin cohort } \\
(n=2838)\end{array}$ & $\begin{array}{l}\text { Total } \\
(N=3056)\end{array}$ \\
\hline Age, years, mean (SD) & $70(4.7)$ & $73(5.9)$ & $73(5.8)$ \\
\hline \multicolumn{4}{|l|}{ Gender, $n(\%)$} \\
\hline Female & $115(52.8)$ & $1451(51.1)$ & $1566(51.2)$ \\
\hline Male & $103(47.3)$ & $1387(48.9)$ & $1490(48.8)$ \\
\hline \multicolumn{4}{|l|}{ Race/ethnicity, $n$ (\%) } \\
\hline White & $202(92.7)$ & $2189(77.1)$ & $2391(78.2)$ \\
\hline Black & $-{ }^{\mathrm{a}}$ & $403(14.2)$ & $412(13.5)$ \\
\hline Hispanic & $-{ }^{\mathrm{a}}$ & $104(3.7)$ & $108(3.5)$ \\
\hline Other & $-{ }^{\mathrm{a}}$ & $142(5.0)$ & $145(4.7)$ \\
\hline Deyo-CC Index, $n$ (\%) & $1.7(1.5)$ & $2.3(1.9)$ & $2.3(1.9)$ \\
\hline DCSI, mean (SD) & $0.7(1.3)$ & $1.3(1.6)$ & $1.2(1.6)$ \\
\hline \multicolumn{4}{|l|}{ Comorbidities, $n(\%)$} \\
\hline Cardiovascular disease & $26(11.9)$ & $755(26.6)$ & $781(25.6)$ \\
\hline Nephropathy & $29(13.3)$ & $754(26.6)$ & $783(26.6)$ \\
\hline Retinopathy & $--^{a}$ & $116(4.1)$ & $124(4.1)$ \\
\hline Peripheral vascular disease & $13(6.0)$ & $249(8.8)$ & $262(8.6)$ \\
\hline Cerebrovascular disease & $--^{\mathrm{a}}$ & $94(3.3)$ & $98(3.2)$ \\
\hline Neuropathy & $29(13.3)$ & $444(15.6)$ & $473(15.5)$ \\
\hline Metabolic disease & $-{ }^{a}$ & $-{ }^{a}$ & $-{ }^{a}$ \\
\hline Obesity & $62(28.4)$ & $445(15.7)$ & $507(16.6)$ \\
\hline Hypoglycemia & $--^{a}$ & $132(4.65)$ & $141(4.6)$ \\
\hline Pre-index prescription fill, counts, mean (SD) & $13.6(8.0)$ & $14.7(8.3)$ & $14.6(8.3)$ \\
\hline \multicolumn{4}{|l|}{ Pre-index antidiabetic medications, $n(\%)$} \\
\hline Biguanides & $166(76.2)$ & $2062(72.7)$ & $2228(72.9)$ \\
\hline Sulfonylureas & $130(59.6)$ & $1811(63.8)$ & $1941(63.5)$ \\
\hline Thiazolidinediones & $39(17.9)$ & $489(17.2)$ & $528(17.3)$ \\
\hline Other antidiabetic medication & $-{ }^{a}$ & $70(2.5)$ & $76(2.5)$ \\
\hline \multicolumn{4}{|l|}{ Pre-index level of antidiabetic therapy, $n(\%)$} \\
\hline No medication use & $12(5.5)$ & $217(7.7)$ & $229(7.5)$ \\
\hline 1 non-insulin antidiabetic & $100(45.9)$ & $1211(42.7)$ & $1311(42.9)$ \\
\hline 2 non-insulin antidiabetics & $87(39.9)$ & $1221(43.0)$ & $1308(42.8)$ \\
\hline$\geq 3$ non-insulin antidiabetics & $19(8.7)$ & $189(6.7)$ & $208(6.8)$ \\
\hline
\end{tabular}


Table 2 continued

\begin{tabular}{|c|c|c|c|}
\hline Characteristic & $\begin{array}{l}\text { Liraglutide cohort } \\
(n=218)\end{array}$ & $\begin{array}{l}\text { Sitagliptin cohort } \\
(n=2838)\end{array}$ & $\begin{array}{l}\text { Total } \\
(N=3056)\end{array}$ \\
\hline Pre-index $\mathrm{HbA} 1_{\mathcal{c}}$, mean $(\mathrm{SD})$ & $8.0(1.4)$ & $7.8(1.4)$ & $7.8(1.4)$ \\
\hline \multicolumn{4}{|l|}{ Baseline glycemic control, $n(\%)$} \\
\hline Controlled: $\mathrm{HbAl}_{\mathrm{c}}<7.0 \%$ & $44(20.2)$ & $770(27.1)$ & $814(26.6)$ \\
\hline $\begin{array}{l}\text { Less strictly controlled: } \mathrm{HbAl}_{\mathrm{c}} \geq 7.0 \% \\
\text { but }<8.0 \%\end{array}$ & $73(33.5)$ & $1038(36.6)$ & $1111(36.4)$ \\
\hline Uncontrolled: $\mathrm{HbAl}_{\mathrm{c}} \geq 8.0 \%$ but $<9.0 \%$ & $55(25.2)$ & $597(21.0)$ & $652(21.3)$ \\
\hline Severely uncontrolled: $\mathrm{HbAl}_{\mathrm{c}} \geq 9.0 \%$ & $46(21.1)$ & $433(15.3)$ & $479(15.7)$ \\
\hline \multicolumn{4}{|l|}{ Prescribing physician specialty, $n$ (\%) } \\
\hline Primary care & $91(41.7)$ & $1094(38.6)$ & $1185(38.8)$ \\
\hline Endocrinology & $65(29.8)$ & $1299(45.8)$ & $1364(44.6)$ \\
\hline Internal and family medicine & $31(14.2)$ & $155(5.5)$ & $186(6.1)$ \\
\hline Other & $40(18.4)$ & $349(12.3)$ & $389(12.7)$ \\
\hline
\end{tabular}

DCSI Diabetes Complications Severity Index, Deyo-CC Index Deyo-Charlson Comorbidity Index, $H b A I_{c}$ glycosylated hemoglobin, $S D$ standard deviation

a Data suppressed to protect privacy

\section{Descriptive Analysis}

Patient demographics and baseline characteristics of each cohort are shown in Table 2. The liraglutide treatment group had a lower mean age (70 vs. 73 years), a lower mean DCSI score (0.7 vs. 1.3$)$, a higher prevalence of obesity ( 28.4 vs. $15.7 \%)$, and a lower prevalence of cardiovascular disease $(11.9$ vs. $26.6 \%)$ and nephropathy (13.3 vs. $26.6 \%)$ than did the sitagliptin treatment group. In addition, the liraglutide treatment cohort had a greater prevalence of uncontrolled $\left(\mathrm{HbA} 1_{\mathrm{c}} \geq 8\right.$ but $\left.<9\right)$ or severely uncontrolled $\left(\mathrm{HbA} 1_{c} \geq 9\right) \quad \mathrm{HbA} 1_{c}$ compared to the sitagliptin treatment group ( 46.3 vs. $36.3 \%$, respectively). The former were also more likely than the sitagliptin treatment group to have received their prescription from an internal medicine or family medicine physician (14.2 vs. $5.5 \%)$ and less likely to have received their prescription from an endocrinologist (29.8 vs. $45.8 \%)$. Overall, a majority $(72.6 \%)$ of patients were from the southern USA, and $60.1 \%$ were enrolled in a health maintenance organization-type insurance plan. Post-index date, insulin was added to the therapy of $5.1 \%$ of patients in the liraglutide group and $8.0 \%$ of those in the sitagliptin group.

The descriptive analysis included all primary and secondary outcome measures. Patients who received liraglutide compared with those who received sitagliptin exhibited a significantly greater decrease in mean $\mathrm{HbA} 1_{c}$ after 1 year of follow-up $(-0.82$ vs. $-0.42 ; \quad P \quad<0.0001)$ (Table 3). The proportion of patients achieving the treatment goal of $\mathrm{HbA} 1_{\mathrm{c}}<7 \%$ was also significantly higher in the liraglutide group than in the sitagliptin group (51.8 vs. $42.1 \%$; $P=0.0052)$. Similarly, the proportion of patients achieving the treatment goal of a reduction in $\mathrm{HbA} 1_{\mathrm{c}} \geq 1 \%$ was significantly higher in the liraglutide group than in the sitagliptin group (40.4 vs. $27.3 \% ; P<0.0001)$. Large proportions of both treatment groups achieved the endpoint treatment goal of $\mathrm{HbA} 1_{\mathrm{c}}<8 \%$ (liraglutide group 78.4\%; sitagliptin group $76.2 \%$ ). No significant total cost difference was observed between liraglutide and 
Table 3 Descriptive analysis of glycemic control and total healthcare costs at 1 year in the inverse probability of treatment weighting sample

\begin{tabular}{|c|c|c|c|}
\hline Glycemic control and total healthcare costs & $\begin{array}{l}\text { Liraglutide cohort } \\
(n=218)\end{array}$ & $\begin{array}{l}\text { Sitagliptin cohort } \\
(n=2838)\end{array}$ & $P$ value \\
\hline Change in $\mathrm{HbA} 1_{c}$, mean $(\mathrm{SD})$ & $-0.82(1.46)$ & $-0.42(1.34)$ & $<0.0001^{*}$ \\
\hline \multicolumn{4}{|l|}{ Patients reaching treatment goals, $n$ (\%) } \\
\hline $\mathrm{HbAl}_{\mathrm{c}}<7 \%$ & $113(51.8)$ & $1195(42.1)$ & $0.0052^{*}$ \\
\hline $\mathrm{HbAl}_{\mathrm{c}}<8 \%$ & $171(78.4)$ & $2163(76.2)$ & 0.4562 \\
\hline Reduction in $\mathrm{HbAl}_{\mathrm{c}} \geq 1 \%$ & $88(40.4)$ & $774(27.3)$ & $<0.0001^{*}$ \\
\hline \multicolumn{4}{|c|}{ Total costs (medical and pharmacy combined costs) of reaching treatment goals, USD, median (IQR) } \\
\hline $\mathrm{HbAl}_{\mathrm{c}}<7 \%$ & $10,248(7560-14,715)$ & $9014(6452-14,531)$ & 0.0936 \\
\hline $\mathrm{HbAl}_{\mathrm{c}}<8 \%$ & $10,514(7350-15,307)$ & $8774(6171-13,863)$ & $0.001^{*}$ \\
\hline
\end{tabular}

Change in $\mathrm{HbAl}_{\mathrm{c}}$ was analyzed using the $t$ test; proportions of patients achieving treatment goals were analyzed using the Chi-square test; costs were analyzed using the Wilcoxon rank sum test

$I Q R$ Interquartile range, USD US dollars

*Statistically significant difference at $P<0.05$

Table 4 Estimated outcomes of glycemic control among patients achieving treatment goal at 1 year in the inverse probability of treatment weighting sample

\begin{tabular}{|c|c|c|c|c|c|}
\hline $\begin{array}{l}\text { Glycemic control } \\
\text { outcomes }\end{array}$ & Treatment & Unadjusted estimates & $P$ value & Adjusted estimates $^{\mathrm{a}}$ & $P$ value \\
\hline $\begin{array}{l}\text { Mean change in } \mathrm{HbAl}_{\mathrm{c}} \\
\quad(95 \% \mathrm{CI})\end{array}$ & $\begin{array}{l}\text { Sitagliptin } \\
\text { Liraglutide }\end{array}$ & $\begin{array}{l}-0.42(-0.47 \text { to }-0.37) \\
-0.82(-1.00 \text { to }-0.64)\end{array}$ & $<0.0001^{*}$ & $\begin{array}{l}-0.12(-0.28 \text { to }-0.04) \\
-0.42(-0.66 \text { to }-0.19)\end{array}$ & $0.0012^{*}$ \\
\hline \multicolumn{6}{|c|}{ Patients reaching treatment goals, odds ratio $(95 \% \mathrm{CI})$} \\
\hline $\mathrm{HbAl}_{\mathrm{c}}<7 \%$ & $\begin{array}{l}\text { Sitagliptin } \\
\text { Liraglutide }\end{array}$ & $\begin{array}{l}1.00 \\
1.48(1.12-1.95)\end{array}$ & $0.0054^{*}$ & $\begin{array}{l}1.00 \\
1.68(1.25-2.24)\end{array}$ & $0.0005^{*}$ \\
\hline $\mathrm{HbAl}_{\mathrm{c}}<8 \%$ & $\begin{array}{l}\text { Sitagliptin } \\
\text { Liraglutide }\end{array}$ & $\begin{array}{l}1.00 \\
1.14(0.81-1.59)\end{array}$ & 0.4564 & $\begin{array}{l}1.00 \\
1.26(0.88-1.80)\end{array}$ & 0.2153 \\
\hline $\begin{array}{l}\text { Mean reduction in } \\
\mathrm{HbA1} 1_{\mathrm{c}} \geq 1 \%\end{array}$ & $\begin{array}{l}\text { Sitagliptin } \\
\text { Liraglutide }\end{array}$ & $\begin{array}{l}1.00 \\
1.81(1.36-2.40)\end{array}$ & $<0.0001^{*}$ & $\begin{array}{l}1.00 \\
1.76(1.31-2.36)\end{array}$ & $0.0002^{*}$ \\
\hline
\end{tabular}

Change in $\mathrm{HbAl}_{\mathrm{c}}$ was analyzed by linear regression; patients achieving treatment goals were analyzed by logistic regression and odds ratios with $95 \%$ confidence interval $(\mathrm{CI})$ is reported

* Statistically significant difference at $P<0.05$

a Models were adjusted for gender, race, health plan type, pre-index level of antidiabetic medication use, post-index oral antidiabetic medication use, and post-index insulin use 
sitagliptin groups achieving an $\mathrm{HbA} 1_{\mathrm{c}}$ of $<7 \%$ prior to IPTW; although the cost of achieving an $\mathrm{HbA}_{\mathrm{c}}$ of $<8 \%$ was significantly higher $(P=$ 0.001) with liraglutide.

\section{Estimated Outcomes of Glycemic Control}

Glycemic control outcomes, estimated using regression models, are presented in Table 4 . The weighted estimated mean decrease in $\mathrm{HbA} 1_{c}$ was greater for patients who received liraglutide than for those who received sitagliptin (estimated difference $-0.40,95 \%$ CI -0.59 to $-0.22 ; P<0.0001$ ), and this difference remained statistically significant clinically when adjusted for covariates (estimated difference $-0.31,95 \%$ CI -0.49 to $-0.12 ; P=$ $0.0012)$. The weighted odds of achieving the treatment goal of $\mathrm{HbA}_{\mathrm{c}}<7 \%$ were 1.48 -fold (95\% CI 1.12-1.95) higher for patients on liraglutide compared with those on sitagliptin $(P=0.0054)$ in the unadjusted analysis, and 1.68-fold (95\% CI 1.25-2.24) higher $(P=$ $0.0005)$ in the adjusted analysis. Additionally, the weighted odds of achieving an $\mathrm{HbA1}_{\mathrm{c}}$ reduction of $\geq 1 \%$ were 1.81 -fold $(95 \% \mathrm{CI}$ 1.36-2.40) higher $(P<0.0001)$ and 1.76-fold (95\% CI 1.31-2.36) higher $(P=0.0002)$ for patients on liraglutide in the unadjusted and adjusted analyses, respectively. Regarding the treatment goal of $\mathrm{HbA}_{\mathrm{c}}<8 \%$, no statistical difference was found between the liraglutide and sitagliptin groups in either the weighted unadjusted or adjusted analyses.

\section{Estimated Total Healthcare Costs}

Total all-cause healthcare costs (medical, pharmacy, outpatient, ED visits, and hospitalization) associated with achievement of the $\mathrm{HbA} 1_{\mathrm{c}}<7 \%$ target were estimated for both the liraglutide and sitagliptin treatment groups. Neither the weighted unadjusted nor adjusted analyses demonstrated statistically significant differences between treatment groups in total healthcare costs for patients achieving a treatment goal of $\mathrm{HbA}_{\mathrm{c}}<7 \%$ (Fig. 3), although total healthcare costs were slightly higher in the liraglutide treatment group. No difference in

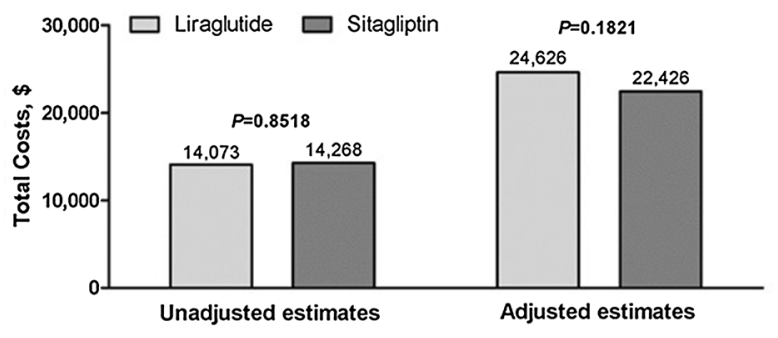

Fig. 3 Estimated unadjusted and inverse probability of treatment weighting adjusted total costs (medical and pharmacy combined) of achieving a $\mathrm{HbAl}_{\mathrm{c}}$ level of $<7 \%$. Numbers above the bars represent the total costs in US dollars

total healthcare costs between treatment groups was found among individuals achieving the treatment goal of $\mathrm{HbA}_{\mathrm{c}}<8 \%$ in the unadjusted analysis; however, when adjusted for post-index antidiabetic treatment, the total healthcare cost was 1.25-fold higher in the liraglutide group (US $\$ 23,088$ vs. US $\$ 18,445$; $P<0.0001)$.

\section{DISCUSSION}

After adjusting for baseline characteristics, the results of this study suggest that in an older population, improved glycemic control (greater mean decrease in $\mathrm{HbA}_{\mathrm{c}}$ and increased likelihood of achieving glycemic treatment goals $\left[\mathrm{HbA} 1_{\mathrm{c}}<7 \%, \mathrm{HbA} 1_{\mathrm{c}}\right.$ reduction of $\left.\geq 1 \%\right]$ ) was associated more with the use of liraglutide than with the use of sitagliptin. All-cause healthcare costs related to achieving an $\mathrm{HbA} 1_{\mathrm{c}}<7 \%$ were slightly higher in the liraglutide group compared to the sitagliptin group, but this difference did not reach statistical significance. This lack of significant difference could be the result of the small sample size.

Although no significant difference was detected between the liraglutide and sitagliptin treatment groups in rates of achieving an $\mathrm{HbA} 1_{c}$ level of $<8 \%$, most patients in both groups (i.e., $>75 \%$ ) achieved this less stringent endpoint. Additionally, the majority of patients in each treatment group had a baseline $\mathrm{HbA} 1_{\mathrm{c}}$ level of $<8.0 \%$, suggesting that a lower treatment goal (e.g., 7.0\%) may have been considered the most appropriate for many of the study 
participants. According to T2D treatment guidelines established by the American Diabetes Association, a reasonable $\mathrm{HbA} 1_{\mathrm{c}}$ goal for many adults without an increased risk for hypoglycemia or other adverse effects of treatment is $<7 \%$, even in an older T2D patient population who are otherwise healthy and should have low glycemic goals [19]. The relatively small prevalence of comorbidities observed in the liraglutide patient population therefore supports the relevance of a lower $\mathrm{HbA} 1_{c}$ goal and our focus on outcomes associated with achievement of an $\mathrm{HbA}_{\mathrm{c}}<7.0 \%$ treatment goal.

GLP-1 RAs and DPP-4 inhibitors are recommended as components of diabetes therapy [20], with GLP-1 RAs reported to have superior glycemic efficacy, pharmacokinetics, and physiologic activity [21]. Compared to the DDP-4 sitagliptin, the GLP1-RA liraglutide may also provide improved cardiovascular safety risk and a reduction in body weight in an older population $[2,22]$. In a clinical trial, compared to a placebo, liraglutide significantly reduced cardiovascular risk factors, including weight, blood pressure, and heart rate. In addition, patients taking liraglutide had a lower risk of nonfatal myocardial infarction, nonfatal stroke, and first occurrence of cardiovascular death compared to the placebo group [22]. In our analysis, the proportion of patients with cardiovascular disease was higher in the sitagliptin group than in the liraglutide group. Consideration of these factors may therefore be of key importance to clinicians, patients, and decision-makers interested in improving outcomes and managing costs associated with T2D.

These data are also supported by similar studies demonstrating superior efficacy and cost-effectiveness of liraglutide versus sitagliptin in the general adult population with T2D $[6,7,12]$. Specifically, the NN2211-1860 (-LIRADPP-4) trial comparing the efficacy and safety of liraglutide versus sitagliptin demonstrated a greater lowering of $\mathrm{HbA} 1_{\mathrm{c}}$ after 26 weeks and 52 weeks of treatment with liraglutide [6, 7]. Additionally, multiple observational studies have confirmed greater reductions in $H b A 1_{c}$ and a higher likelihood of achieving glycemic endpoints with liraglutide versus sitagliptin during a 6-month assessment [8-11]. A recent real-world study has also highlighted the longterm effectiveness of liraglutide in this population [12]. A meta-analysis also demonstrated the efficacy and safety of liraglutide compared with sitagliptin when combined with metformin [23]. Furthermore, various studies sourcing data from clinical trials and claims data demonstrated better cost-effectiveness of liraglutide compared with sitagliptin, with any increases in pharmacy costs associated with liraglutide being offset by decreases in other diabetes-related medical expenses [13-16].

\section{LIMITATIONS}

This analysis may be limited by the inconsistency in data collection processes inherent in claims data, including the absence of available disease severity information, an important prognostic factor in determining treatment outcomes. However, IPTW methodology was incorporated as a strategy of mitigating unmeasured confounding. The IPTW methodology may have increased standardized bias; additional sensitivity analysis could quantify any potential residual confounding. Results may also have been influenced by the exclusion of patients with baseline use of insulin, which may have resulted in the selection of individuals with less severe disease and may not be reflective of what is experienced in the realworld clinical setting. The analysis cohort sample size was also limited by the requirement of $\mathrm{HbA} 1_{\mathrm{c}}$ results and persistence on index therapy; however, these data were considered essential in comparing the effects of liraglutide and sitagliptin treatments. Excluding patients that did not have HbA1c values at 1-year post-baseline could have introduced selection bias. Of note, previous clinical studies comparing the efficacy of liraglutide versus sitagliptin included stratification of liraglutide doses, with a higher dose demonstrating greater efficacy [6, 7]; however, assigning the liraglutide dose using claims data would be subject to a lack of patient information, and dosing information was therefore not included in the current analysis. The detection of hypoglycemic events using claims data was 
also limited and potentially restricted to severe events requiring medical intervention, despite the importance of hypoglycemic risk in treating older people with diabetes. This lack of hypoglycemic event data precluded our ability to include 'no hypoglycemia' in composite outcomes. Finally, whereas the study was sufficiently powered for the main outcome, any inference on secondary outcomes (i.e., cost) may not have sufficient sample size and may be subject to type II error. Results reported on secondary outcomes should be used to inform additional research.

\section{CONCLUSIONS}

These real-world data add to a body of evidence that suggests liraglutide is associated with a greater $\mathrm{HbA} 1_{c}$ reduction compared to sitagliptin, and this association is maintained in an older population. Older people with T2D who initiate liraglutide treatment may be more likely to achieve treatment goals of $\mathrm{HbA} 1_{\mathrm{c}}<7 \%$ and an $\mathrm{HbA} 1_{\mathrm{c}}$ reduction of $\geq 1 \%$ after 1 year of therapy compared with those who initiate treatment with sitagliptin. Additionally, cost data offer preliminary evidence that glycemic benefits of liraglutide are not associated with a significant increase in all-cause health care costs compared to sitagliptin, although further longer-term evaluation is warranted.

\section{ACKNOWLEDGEMENTS}

Funding. Humana Comprehensive Health Insights ${ }^{\circledR}$ Inc. received financial support from Novo Nordisk Inc., Plainsboro, New Jersey, to conduct this study. All authors had full access to all of the data in this study and take complete responsibility for the integrity of the data and accuracy of the data analysis. Novo Nordisk Inc. also funded the journal's Rapid Service Fee for this publication.

Authorship. All named authors meet the International Committee of Medical Journal Editors (ICMJE) criteria for authorship for this article, take responsibility for the integrity of the work as a whole, and have given their approval for this version to be published.

Authorship Contributions. All authors were involved in the conception and design and/or analysis and interpretation of the data, and all authors were involved in the drafting of the paper and revising it critically for intellectual content. The authors acknowledge the contributions of Ray Harvey of Humana Healthcare Research.

Medical Writing. Writing assistance was provided by Anna Abt, PhD, of ETHOS Health Communications in Yardley, Pennsylvania, and was supported financially by Novo Nordisk Inc., Plainsboro, New Jersey, in compliance with international Good Publication Practice guidelines.

Disclosures. Tam Dang-Tan is an employee of Novo Nordisk Inc; Cory Gample is an employee of Novo Nordisk Inc; Rahul Ganguly is an employee of Novo Nordisk Inc; Libby Horter is an employee of Humana Healthcare Research (formerly Comprehensive Health Insights ${ }^{\circledR}$ Inc.); Pravin S. Kamble was an employee of Comprehensive Health Insights Inc. at the time of the study; he is currently employed by Sunovion Pharmaceuticals. Yunus Meah is an employee of Humana Inc. An abstract of this study was presented at AMCP Nexus 2017 as a poster presentation, and is published in the Journal of Managed Care \& Specialty Pharmacy: https://www.jmcp.org/pbassets/Poster\%20Abstract\%20Supplements/ Oct2017Abstracts.pdf.

Compliance with Ethics Guidelines. The research protocol associated with the manuscript was reviewed and approved as a minimal risk study by Schulman IRB, an independent institutional review board, which determined that the study met the criteria for a waiver of informed consent and waiver of authorization as set forth by the code of federal regulations.

Data Availability. The datasets generated during and/or analyzed during the current study are available from the corresponding author on reasonable request. 
Open Access. This article is distributed under the terms of the Creative Commons Attribution-NonCommercial 4.0 International License (http://creativecommons.org/licenses/ by-nc/4.0/), which permits any noncommercial use, distribution, and reproduction in any medium, provided you give appropriate credit to the original author(s) and the source, provide a link to the Creative Commons license, and indicate if changes were made.

\section{APPENDIX 1}

See Table 5.

Table 5 Deyo-Charlson comorbidity index, related ICD-9-CM codes, and weighting

\begin{tabular}{|c|c|c|}
\hline Comorbidity & ICD-9-CM codes & Weight \\
\hline Myocardial infarction & 410.xx, 412.xx & 1 \\
\hline Congestive heart failure & $428 . x x$ & 1 \\
\hline Peripheral vascular disease & 441.xx, 443.9, 785.4, V43.4, 38.48* & 1 \\
\hline Cerebrovascular disease & 430.xx-437.xx, 438.xx & 1 \\
\hline Dementia & 290.xx & 1 \\
\hline Chronic pulmonary disease & 490.xx-496.xx, 500.xx-505.xx, 506.4 & 1 \\
\hline Connective tissue disease & 710.xx, 714.xx, 725.xx & 1 \\
\hline Peptic ulcer disease & $\begin{array}{l}531.4 x-531.7 x, 532.4 x-532.7 x, 533.4 x-533.7 x, 534.4 x-534.7 x, 531.0 x-531.3 x \\
532.0 x-532.3 x, 533.0 x-533.3 x, 534.0 x-534.3 x, 531.9 x, 532.9 x, 533.9 x, 534.9 x\end{array}$ & 1 \\
\hline Mild liver disease & $571.2,571.4,571.5,571.6$ & 1 \\
\hline $\begin{array}{l}\text { Diabetes without } \\
\text { complications }\end{array}$ & $250.0 x-250.3 x, 250.7 x$ & 1 \\
\hline Diabetes with complications & $250.4 x-250.6 x$ & 2 \\
\hline Paraplegia and hemiplegia & 342.x, 344.1 & 2 \\
\hline Renal disease & 582.x, 583.0-583.7, 585.xx, 586.xx, 588.xx & 2 \\
\hline $\begin{array}{l}\text { Cancer (including leukemia } \\
\text { and lymphoma) }\end{array}$ & 140.xx-172.xx, 174.xx-195.xx, 200.xx-208.xx & 2 \\
\hline $\begin{array}{l}\text { Moderate or severe liver } \\
\text { disease }\end{array}$ & $572.2-572.8$ & 3 \\
\hline Metastatic carcinoma & $196 . x-199 . x$ & 6 \\
\hline $\begin{array}{l}\text { Acquired immunodeficiency } \\
\text { syndrome (AIDS) }\end{array}$ & 042.xx-044.x & 6 \\
\hline
\end{tabular}

ICD-9-CM International Classification of Diseases, Ninth Revision, Clinical Modification 


\section{REFERENCES}

1. Nunes AP, Yang J, Radican L, et al. Assessing occurrence of hypoglycemia and its severity from electronic health records of patients with type 2 diabetes mellitus. Diabetes Res Clin Pract. 2016;121:192-203.

2. Hepburn DA, Deary IJ, Frier BM, Patrick AW, Quinn JD, Fisher BM. Symptoms of acute insulin-induced hypoglycemia in humans with and without IDDM. Factor-analysis approach. Diabetes Care. 1991;14(11):949-57.

3. Corriere M, Rooparinesingh N, Kalyani RR. Epidemiology of diabetes and diabetes complications in the elderly: an emerging public health burden. Curr Diabetes Rep. 2013;13(6):805-13.

4. Kirkman MS, Briscoe VJ, Clark N, et al. Diabetes in older adults. Diabetes Care. 2012;35(12):2650-64.

5. Nauck MA. A critical analysis of the clinical use of incretin-based therapies: the benefits by far outweigh the potential risks. Diabetes Care. 2013;36(7):2126-32.

6. Pratley R, Nauck M, Bailey $T$, et al. One year of liraglutide treatment offers sustained and more effective glycaemic control and weight reduction compared with sitagliptin, both in combination with metformin, in patients with type 2 diabetes: a randomised, parallel-group, open-label trial. Int J Clin Pract. 2011;65(4):397-407.

7. Pratley RE, Nauck M, Bailey T, et al. Liraglutide versus sitagliptin for patients with type 2 diabetes who did not have adequate glycaemic control with metformin: a 26-week, randomised, parallel-group, open-label trial. Lancet. 2010;375(9724):1447-56.

8. Lee WC, Dekoven M, Bouchard J, Massoudi M, Langer J. Improved real-world glycaemic outcomes with liraglutide versus other incretin-based therapies in type 2 diabetes. Diabetes Obes Metab. 2014;16(9):819-26.

9. Li Q, Chitnis A, Hammer M, Langer J. Real-world clinical and economic outcomes of liraglutide versus sitagliptin in patients with type 2 diabetes mellitus in the United States. Diabetes Ther. 2014;5(2):579-90.

10. Lind $\mathrm{M}$, Matsson PO, Linder $\mathrm{R}$, et al. Clinical effectiveness of liraglutide vs sitagliptin on glycemic control and body weight in patients with type 2 diabetes: a retrospective assessment in Sweden. Diabetes Ther. 2016;7(2):321-33.

11. Nyeland ME, Ploug UJ, Richards A, et al. Evaluation of the effectiveness of liraglutide and sitagliptin in type 2 diabetes: a retrospective study in UK primary care. Int J Clin Pract. 2015;69(3):281-91.

12. Lapolla A, Berra C, Boemi M, et al. Long-term effectiveness of liraglutide for treatment of type 2 diabetes in a real-life setting: a 24-month, multicenter, non-interventional, retrospective study. Adv Ther. 2018;35(2):243-53.

13. Davies MJ, Chubb BD, Smith IC, Valentine WJ. Cost-utility analysis of liraglutide compared with sulphonylurea or sitagliptin, all as add-on to metformin monotherapy in type 2 diabetes mellitus. Diabet Med. 2012;29(3):313-20.

14. DeKoven M, Lee WC, Bouchard J, Massoudi M, Langer J. Real-world cost-effectiveness: lower cost of treating patients to glycemic goal with liraglutide versus exenatide. Adv Ther. 2014;31(2):202-16.

15. Langer J, Hunt B, Valentine WJ. Evaluating the short-term cost-effectiveness of liraglutide versus sitagliptin in patients with type 2 diabetes failing metformin monotherapy in the United States. J Manag Care Pharm. 2013;19(3):237-46.

16. Tzanetakos C, Melidonis A, Verras C, Kourlaba G, Maniadakis N. Cost-effectiveness analysis of liraglutide versus sitagliptin or exenatide in patients with inadequately controlled type 2 diabetes on oral antidiabetic drugs in Greece. BMC Health Serv Res. 2014;14:419. https://doi.org/10.1186/4726963-14-419.

17. United States Department of Labor, Bureau of Labor Statistics. Consumer Price Index. http://www.bls. gov/cpi/. Accessed 15 July 2019.

18. Austin PC, Stuart EA. Moving towards best practice when using inverse probability of treatment weighting (IPTW) using the propensity score to estimate causal treatment effects in observational studies. Stat Med. 2015;34(28):3661-79.

19. Professional Practice Committee. Standards of medical care in diabetes-2018. Diabetes Care. 2018;41 Suppl 1:S3.

20. Bailey T. Options for combination therapy in type 2 diabetes: comparison of the ADA/EASD position statement and AACE/ACE algorithm. Am J Med. 2013;126 9 Suppl 1:S10-20.

21. Nauck M. Incretin therapies: highlighting common features and differences in the modes of action of glucagon-like peptide-1 receptor agonists and dipeptidyl peptidase-4 inhibitors. Diabetes Obes Metab. 2016;18(3):203-16.

22. Marso SP, Daniels GH, Brown-Frandsen $\mathrm{K}$, et al. Liraglutide and cardiovascular outcomes in type 2 diabetes. N Engl J Med. 2016;375(4):311-22. 
23. Li M, Yang Y, Jiang D, Ying M, Wang Y, Zhao R. Efficacy and safety of liraglutide versus sitagliptin both in combination with metformin in patients with type 2 diabetes: a systematic review and metaanalysis. Medicine. 2017;96(39):e8161. 